\title{
Medical abortion: the hidden revolution
}

\author{
Phil Harvey
}

Chairman, DKT International, Washington DC, USA

\section{Correspondence to} Mr Phil Harvey, DKT International, 1701K Street NW, Suite 900, Washington DC 20006, USA; phil@dktinternational.org

Received 29 May 2014 Revised 27 March 2015 Accepted 20 April 2015

\section{CrossMark}

To cite: Harvey P. J Fam Plann Reprod Health Care 2015;41:193-196.

\section{ABSTRACT}

While the medical abortion (MA) drugs, mifepristone and misoprostol, have radically altered reproductive health practices around the world, there has been little field research on the sales and use of these drugs, especially in developing countries. This leaves the family planning community with many unanswered questions. While good profiles of contraceptive use are available for many countries and we have good technical data on the MA drugs' efficacy, dosages and regimens such as home dosage of misoprostol versus clinic dosage, we have very little information about the quantities of MA drugs sold, how they are used, where they are used, and, in the case of misoprostol, for what purposes. Sales data are available from one excellent commercial survey and from social marketing sales of mifepristone and misoprostol and these are presented. Acknowledging the sensitivity of the issue, especially in countries where abortion is severely restricted, the author makes a plea for careful additional research to shed light on an important and growing part of the international reproductive health picture.

\section{INTRODUCTION}

The two drugs used for early termination of pregnancy, mifepristone and misoprostol, may well have altered fertility patterns in the developing world more than any other reproductive health intervention in the last two or three decades.

Despite the unquestionable importance of these medical abortion (MA) drugs, we have very little information from the developing world about how many are being used, by whom, or where.

The reason is, of course, because MA, probably even more than surgical abortion, is so often performed clandestinely. Because of laws and cultural norms restricting abortions in most developing countries, MA procedures are not reported in those countries in any systematic way. Indeed it is likely that a substantial portion of such procedures are never

\section{Key message points}

Medical abortion (MA), using mifepristone and/or misoprostol, has had a dramatic impact on the reproductive lives of couples and individuals residing in developing countries, yet we know very little about how these drugs are used.

- The inclusion of a few simple questions about pregnancy termination in the Demographic and Health Surveys could provide valuable new information on the impact of MA drugs.

- Estimating the number of abortions from misoprostol sales is complicated; however, educated estimates suggest that $20-40 \%$ of abortions in developing countries are performed in this way.

reported at all. Thus, while a great deal of clinical research on the efficacy of these drugs has been published, and we know a lot about appropriate dosages, effective gestational dates, and modes of application, ${ }^{1-3}$ we are groping in the dark when it comes to quantifying the extent of MA's impact on reproductive health and fertility patterns in much of the developing world. To pick a random example, we have a pretty good idea from Demographic and Health Survey (DHS) data of how many women in Malawi use injectable contraceptives (about 540000 ), but no information at all about the contribution made by MA to reproductive health in that, or most other, low-income countries.

This article will summarise what we do know, estimating the number of likely procedures from sales data where available, take a brief look at why it would be helpful to know more, and address a few of the problems that might arise from seeking that information. 


\section{BACKGROUND}

Mifepristone is an antiprogestogen that was developed by Roussel Uclaf Labs in France and released in 1988, and is commonly known as the 'abortion pill' or RU-486. It is included in the World Health Organization's (WHO's) Model List of Essential Medicines without usage recommendations ("where permitted under national law and where culturally acceptable"), despite some urging from the medical community that it be listed as an abortifacient. ${ }^{4}$ However, WHO's policy guidelines include Safe Abortion: Technical and Policy Guidance for Health Systems, ${ }^{5}$ which clearly recommends both mifepristone and misoprostol for early abortion.

Misoprostol is a prostaglandin that was approved by the U.S. Food and Drug Administration in 1988 as a preventative for gastric ulcers and is listed in WHO's Model List of Essential Medicines for use as an oxytocic to prevent postpartum haemorrhage, for postabortion care $(200 \mu \mathrm{g})$, and "where appropriate" for induction of labour $(25 \mu \mathrm{g})$. As already noted, this drug is also listed as an abortifacient in the WHO Safe Abortion guideline.

MA has several advantages over surgical abortion, perhaps even more markedly in developing countries than in the West. Where access to clinics and hospitals is limited, women who are desperate to end a pregnancy can do so safely (overdosing is virtually impossible with misoprostol and mifepristone) instead of resorting to the still common practices of ingesting poisons, undergoing violent abdominal massages, or probing the uterus with sticks or wires that can cause serious injuries, bleeding or infection and are all too often fatal.

While a MA is not without pain and bleeding, it is not dangerous, and women who use these drugs report high levels of satisfaction with the procedure. ${ }^{6}$ One review of the literature found that "In most studies $80 \%$ of women who chose medical abortion found it acceptable and would choose the same method again if they needed another abortion in the future". ${ }^{7}$ And, while abortion is not normally recommended as a regular method of fertility control, MA offers the main health advantages of birth spacing: lower maternal mortality and morbidity, and lower infant death rates. Indeed the rapid drop in maternal mortality in Latin America in recent years may be partly due to the use of misoprostol for post-abortion care, a treatment that is generally legal even where most or all abortions are not.

Unlike surgical abortion, which requires a trained practitioner (normally a doctor or midwife), MA involves only taking specified doses of specified drugs. This means that pharmacists in some countries can sell the drugs and provide the needed information, assuming that they have it. Where the drugs are available over-the-counter they may be especially convenient and, if accompanied by proper instructions, they are both safe and effective. MA is low-cost in most low-income countries, where these medicaments can normally be purchased for substantially less than a surgical procedure. A MA 'kit' in India (normally one $200 \mathrm{mg}$ mifepristone tablet + four $200 \mu \mathrm{g}$ tablets of misoprostol) cost consumers about 500 rupees, less than US\$10, in 2014. Misoprostol tablets $(200 \mu \mathrm{g})$ can be purchased for less (often much less) than US $\$ 1$ in most of the world's low-income countries.

\section{HOW MUCH MA IS SOLD?}

While there is not a great deal of sales data on MA drugs, there is some. One useful article $^{8}$ has aggregated data on misoprostol sales from IMS Health, a firm that publishes international pharmaceutical sales data. It reports total developing country sales of 79 million misoprostol tablets in 2007. We do not know how many abortions resulted from these sales, but a reasonable estimate is roughly 5 million. The only organisations that make these calculations, Marie Stopes International and DKT International, non-profit organisations that promote family planning, have arrived at a rough conversion factor of 16 misoprostol tablets per abortion, based on the fact that 8-12 tablets are needed to induce an abortion (when used without mifepristone), and that a substantial fraction of reported misoprostol sales are used for non-abortion purposes. This estimate is admittedly very crude. WHO's Safe Abortion Paper, like most other MA protocols, calls for $200 \mathrm{mg}$ mifepristone followed by four $200 \mu \mathrm{g}$ misoprostol tablets a day or two later. For misoprostol used alone, the recommended dosage is four tablets, with up to three more doses of four tablets each at 3-hourly intervals if needed. So there is considerable variation in the number of tablets needed to complete an abortion with misoprostol alone, and the conversion factor can only be an estimate.

The estimate of 5 million abortions from the IMS Health data may be too low for a different reason. The authors of the paper were careful to note that 79 million tablets were official sales of registered brands and that "The black market is believed to play an important role in the [Latin American] region", so actual sales could have been substantially more. Still, the data seem reasonable. The review reports sales of 13 million registered-brand tablets in Latin America in 2007, for example, enough for nearly 1 million abortions or roughly one-quarter of the estimated annual abortions in that area. ${ }^{9}$

We also have some limited sales data from social marketing organisations, which have recently added the MA drugs to their marketing portfolios. ${ }^{10}$ Social Marketing managers in 65 countries reported contraceptive sales in 2012. Fifteen of these countries (Burma, Cambodia, Egypt, Ethiopia, Ghana, Guatemala, India, Indonesia, Mexico, Mozambique, Nepal, Sudan, Tanzania, Uganda, Vietnam) reported sales of MA and/or misoprostol tablets. Of these, eight sold misoprostol only, and the rest sold MA kits and, in some cases, 
misoprostol tablets as well. The seven countries that included MA kits were Cambodia, Ethiopia, Ghana, India, Nepal, Uganda and Vietnam. Sales of these kits, comprised of one $200 \mathrm{mg}$ tablet of mifepristone and four $200 \mu \mathrm{g}$ tablets of misoprostol, rose from 1375000 in 2010 to 1915000 in 2011 and 1840000 in 2012. Excluding India (a special case - see below), these figures suggest that MA pills, sold through social marketing, accounted for about one-third of estimated abortions in those six countries.

Social marketing sales of misoprostol tablets without mifepristone were added to the social marketing statistical reports starting in 2011, when sales of 13.2 million tablets were reported from 13 country programmes, with the largest number in Egypt. Sales of misoprostol continued upwards in 2012, to 16.9 million tablets in 12 countries. Total estimated abortions in those countries were estimated at 5.7 million (again excluding India), meaning that the tablets sold through social marketing, and using the conversion estimate of 16 tablets per abortion, probably provided about 1 million abortions or $18 \%$ of the total for those 12 countries. The great majority of social marketing sales of MA drugs are through pharmacies.

The sales figures from India present an additional twist on data calculations for MA. The Indian branch of IMS Health, considered a reliable source of pharmaceutical sales statistics in India, reported the sale of 10 million misoprostol tablets there in 2010, which seems reasonable. But the same source reported the sale of 6.3 million mifepristone/misoprostol combination kits in 2011 and 7.7 million in 2012. These combination kits can only be used for abortion as mifepristone is the most expensive component and has very few other uses, and 7 million kit sales exceed the total estimated annual abortions in India, which is obviously unlikely. Explanations for this discrepancy include the possibility that some of these kits are being exported to other countries, which would help spread this method elsewhere; or there could be overstocking of unused inventory, and some wastage due to expiry. But it is also possible that there are many more abortions in India than the official estimate of 6.7 million. ${ }^{11}$ In any event, it suggests that MA has become very popular in India, with major commercial suppliers competing with the social marketing programmes, resulting in considerable publicity about the method, and consequent high levels of sales.

Online sales of MA drugs appear to be negligible, at least so far. One valuable internet service, Women on Waves, has done a great deal to publicise the availability of MA and continues to provide valuable information about its use; however, that service follows legal protocols and sells only about 7000 doses per year.

\section{DO WE NEED TO KNOW MORE?}

For those working in international family planning or health programmes generally - the provision of more information on the use of MA drugs would have real value. We simply do not know if these medications are being used properly. Overdosing with misoprostol, for example, while not fatal, has some serious side effects. FIGO (The International Federation of Gynecology and Obstetrics) revised their dosage guidelines in 2012, lowering the dosages for second-trimester abortions due to concerns about possible uterine rupture at higher doses, especially in cases of previous caesarean sections or vaginal scarring. But even in the first trimester, women may be over- or under-dosing. Mifepristone for years was prescribed in doses of $600 \mathrm{mg}$, much more than necessary, and this protocol is still followed in some places. While the higher dose it not harmful, mifepristone is expensive and the extra unnecessary cost could put the procedure out of reach for many women. We just do not know if women are taking too much of these drugs, or too little.

Another question involves alternative uses. There are several approved uses for misoprostol, but is it being used for purposes beyond those? Mifepristone's uses are very limited, but there are some indications that it is being used as a monthly contraceptive in India, for example. Is this common? Is it safe and effective? It is marketed in Vietnam in $25 \mathrm{mg}$ tablets for emergency contraception, for which doses between 10 and $50 \mathrm{mg}$ have been found to be quite effective. ${ }^{12}$ Pharmacists may be prescribing these low dosages for abortion as well.

It would be very useful to know how many women acquire and use MA drugs in, for example, a country like Ghana. What implications would that have for Ghana's contraceptive prevalence rate? Or Ethiopia's? Are the millions of Indian women, or their partners, getting adequate instructions for the abortion drugs they buy in that country?

\section{THE DANGERS OF LEARNING TOO MUCH}

Research on the uses of drugs that cause abortions of course carries risks. The most immediate is that government authorities, learning of the increasing scale of these drugs' use for pregnancy termination, would feel compelled to restrict them or forbid their import or manufacture. Those who prescribe the drugs might fear arrest or harassment and refuse to sell the drugs. Misoprostol's multiple uses are very helpful in this regard as they allow governments to permit trade in that drug, explicitly for non-abortion uses, while not strictly policing off-label uses. Thus the status quo for misoprostol, including a considerable degree of ignorance about its use, may be acceptable, at least for countries where abortion is severely restricted.

Conversely, inclusion of a few abortion-related questions in the DHS surveys, which have conspicuously omitted this subject so far, would not appear to carry much risk. Every health minister knows there are abortions in their country - legal or illegal - and quantifying such information should help everyone, 
including those who back policies to make contraceptives more readily available. Many 'abortions' are spontaneous and therefore uncontroversial, so a few simple questions should not incur much risk.

In countries where abortions, including MAs, are legal, most notably India, full-blown research on the use and prevalence of the sale of MA drugs is perfectly feasible and should be undertaken. The subject is rarely controversial there and advertisements promoting MA have run on national television ${ }^{13}$ and caused no significant negative reaction. Research should also be feasible in such countries as South Africa, Vietnam, Tunisia and others with liberal abortion policies.

Finally, new research on the sale of these MA drugs, taking advantage of the work done routinely by such companies as IMS Health, will give us all a much better idea of the scope and impact of MA worldwide.

Competing interests None declared.

Provenance and peer review Not commissioned; externally peer reviewed.

\section{REFERENCES}

1 Berer M. Medical abortion: a fact sheet. Reprod Health Matters 2005;13:26.

2 The ICMA Information Package on Medical Abortion. http://www. medicalabortionsoncortium.org/articles/for-women/main book/? $\mathrm{bl}=\mathrm{en}$ and at http://misoprostol.org/ [accessed 26 March 2015].
3 Gynuity Health Projects. http://gynuity.org [accessed 26 March 2015].

4 Abortion drugs must become WHO essential medicines. Lancet 2005;365:1826.

5 World Health Organization. Safe Abortion: Technical and Policy Guidance for Health Systems (2nd edn). 2013. http:// www.who.int/reproductivehealth/publications/unsafe_abortion/ 9789241548434/en/ [accessed 26 March 2015].

6 Berer M. Medical abortion: issues of choice and acceptability. Reprod Health Matters 2005;13:26.

7 Ho PC. Women's perceptions on medical abortion. Contraception 2006;74:11-15.

8 Fernandez MM, Coeytaux F, de León RG, et al. Assessing the global availability of misoprostol. Int J Gynaecol Obstet 2009;105:180-186.

9 Sedgh G, Singh S, Shah IH, et al. Induced abortion: incidence and trends worldwide from 1995 to 2008. Lancet 2012;379:625-632.

10 DKT International. Contraceptive Social Marketing Statistics (annual data).

11 Chhabra R, Nuna SC. Abortion in India: An Overview. 1994. http://www.cehat.org/publications/PDF\%20files/r24.pdf [accessed 26 March 2015].

12 Mittal S, Aggarwal P. Interventions for Emergency Contraception: RHL Commentary (last revised: 1 November 2012). The WHO Reproductive Health Library. Geneva, Switzerland: World Health Organization, 2012.

13 Television advertisement for medical abortion pills from DKT India 'A Doctor Tells a Doctor'. http://www.youtube.com/ watch? $\mathrm{v}=\mathrm{wEzW} 8 \mathrm{ijXeCw}$ [accessed 26 March 2015].

\title{
Menopause Theory Course (FSRH Menopause Special Skills)
}

\author{
12th \& 13th November 2015 at Holiday Inn Bristol City Centre, Bristol BS1 3LE
}

This course will be led by Sarah Gray, a GP specialist who runs the Menopause Clinic in Cornwall and is a FSRH Primary Trainer in Menopause. She will be joined by experienced clinical colleagues from around the country. They will summarise evidence and discuss its application to clinical practice. A lecture and small group format will allow interaction, case based discussion and build confidence.

The course meets the theory requirement for both basic and advanced special skills certification and sub-speciality training. It would however benefit any doctor or nurse whose clinical workload includes post reproductive health.

\section{3 hours CME \\ Course Fee $£ 395$ (not residential)}

Further details and application form online at www.crescetis.co.uk

Mike Gray

Crescetis

Egloserme Farm

St Erme

Truro

TR4 9BW

Tel: 01872242192

Email: info@crescetis.com 